\title{
Aptamer-based Colorimetric Biosensing of Ochratoxin A in Fortified White Grape Wine Sample Using Unmodified Gold Nanoparticles
}

\author{
Xueting Yin, Sai Wang, Xiaoyun LiU, Chenmeng He, Yali Tang, Qimeng Li, Jiahui LiU, Haijia Su, \\ Tianwei TAN, and Yiyang DonG ${ }^{\dagger}$
}

Beijing Key Laboratory of Bioprocess, College of Life Science and Technology, Beijing University of Chemical Technology, Beijing 100029, P. R. China

\begin{abstract}
In this study, a simple and sensitive aptamer-based colorimetric method for the detection of Ochratoxin A by using gold nanoparticles (AuNPs) has been developed. In this assay, unmodified gold nanoparticles (AuNPs) were used as probes with a 36-mer aptamer as recognition element. In the absence of ochratoxin A, free aptamer could be adsorbed onto the surface of AuNPs and protect AuNPs from aggregation even with high concentrations of salt. The salt-induced aggregation of AuNPs was caused by the specific recognition of aptamers with OTA. Under optimum conditions, calibration modeling showed that the analytical linear range covered from 32 to $1024 \mathrm{ng} / \mathrm{mL}$ and the detection limit of $20 \mathrm{ng} / \mathrm{mL}$ was realized successfully. This proposed colorimetric bio-assay also showed high selectivity over other antibody based methods. Meanwhile, this strategy was further used to determine the concentrations of ochratoxin A in white wine sample with satisfying recovery rates.
\end{abstract}

Keywords Colorimetric detection, gold nanoparticles, aptamers, ochratoxin A, fortified white grape wine

(Received September 12, 2016; Accepted December 14, 2016; Published June 10, 2017)

\section{Introduction}

The contamination of foodstuff and feed by mycotoxins has become an increasingly serious problem. Mycotoxins are new considered a major risk to human and animal health, and ochratoxin A (OTA) is one of the most common mycotoxins. ${ }^{1}$ OTA is a potent fungal toxin produced by Aspergillus ochraceus and Penicillium verrucosum ${ }^{2}$ and it has been reported that OTA is potentially nephrotoxic, carcinogenic, hepato-toxic, teratogenic and immunotoxic to humans with high chemical stability and thermostability. ${ }^{3}$ What makes it worse is that OTA is the most widespread naturally occurring mycotoxin, existing in many food products including cereals, wine, corn, oats, coffee beans, beer, coffee, grape juice and wheat. ${ }^{4}$ A number of sensitive methods have been developed to determine the concentration of OTA in foodstuffs. ${ }^{5}$ The conventional analytical methods for OTA included gaschromatography (GC) or high-performance liquid chromatography (HPLC) coupled with UV-Vis, or mass spectrometric detectors. ${ }^{6}$ The use of instrumental methods is hindered by the use of expensive instruments and time consuming procedures. Moreover, many aptamer-based biosensors (aptasensors) for OTA detection have been reported in recent years, such as enzyme-linked aptamer assays (ELAA) and fluorescent aptasensor. ${ }^{7}$ Compared with aptamers, the preparation of antibodies is complicated and

X. Y. and S. W. contributed equally to this work.

${ }_{\dagger}$ To whom correspondence should be addressed.

E-mail: yydong@mail.buct.edu.cn antibodies may also be susceptible to several processes of sample preparation or condition optimization in the study. ${ }^{2}$ As such, aptamers have been selected as an alternative to antibodies for mycotoxin biosensing.

Recently, aptamers have been increasingly used due to their convenient advantages compared with other biological tools. Aptamers are single-stranded oligonucleotides of DNA or RNA sequences obtained by an in vitro selection process named the systematic evolution of ligands by exponential enrichment (SELEX), which would bind to the surface of gold nanoparticles (AuNPs) through electrostatic interaction and specially forming the individual 3D structures. ${ }^{8,9}$ The selected aptamers were easily obtained by chemical synthesis, which provided several advantages such as low cost, inherent selectivity, high stability, and a greater surface density of receptors on AuNPs. ${ }^{10}$ Compared with aptamers, antibodies are easily affected by temperature and $\mathrm{pH}$ variations and undergo irreversible denaturation, ${ }^{11}$ while aptamers are stable against various variants. ${ }^{12}$ Because on the outstanding characteristic of aptamers, several studies were reported on aptasensors using the colorimetric method for various substrates ${ }^{13}$ including antigen, ${ }^{14}$ copper ions ${ }^{15}$ and adrenaline ${ }^{16}$ with specific spatial structures such as targetinduced folding or formation of double strands. In addition, there have been numerous methods developed based on the aptamer, for example, fluorescence aptamer assay, quartz crystal microbalance, electrochemical systems, and so on. ${ }^{13}$

Nowadays, AuNPs are widely used in immunoassay studies as reporting probes. ${ }^{17,18}$ AuNPs showed high extinction coefficients and distance-dependent optical properties in DNA-related colorimetric assay ${ }^{19}$ and were easily affected by their particle 
stability and inter-particle distance. The dispersed AuNPs were red, while the aggregated AuNPs showed a blue color. This was associated with the phenomena called surface plasmon resonance (SPR). ${ }^{20}$ Furthermore, the SPR was related to the size, shape, and interparticle spacing of the gold nanoparticles, ${ }^{21}$ leading to the AuNPs absorbance and scattering thoroughly compared with those of conventional materials. ${ }^{14}$ With the color changing of the transition of AuNPs, the results can be observed by the naked eye. Many AuNPs-based colorimetric methods have been established to detect various analytes, including protein, drugs, small molecules, inorganic ions and even cells. ${ }^{22}$ Hence, there is an urgent need to develop a superior colorimetric method for analytes.

In this study, we developed a sensitive and convenient labelfree colorimetric detection method for OTA using ssDNA aptamer as recognition element and unmodified gold nanoparticles as probes with an improved linear range and an extra optimization strategy of aptamer-OTA reaction time based on our previous work. ${ }^{23}$ A 36-mer aptamer was used in the study because of its higher recognition than longer aptamers. AuNPs were easily absorbed by uncombined aptamer preventing the aggregation, which made the color remain red. Then the color turned to blue following AuNPs aggregation with $\mathrm{NaCl}$

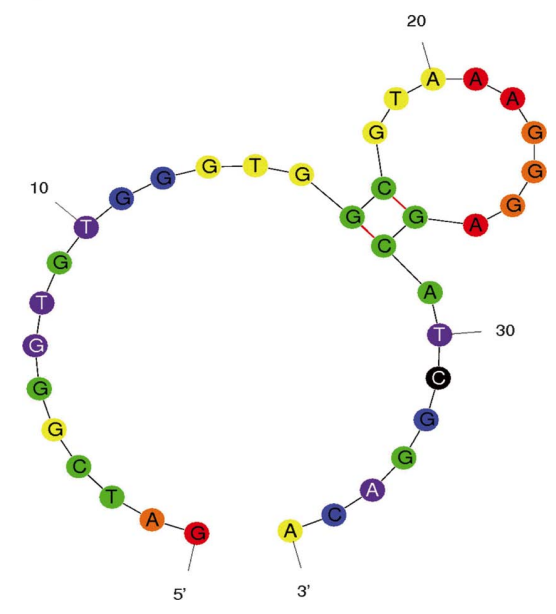

Fig. 1 Secondary structure of the 40-mer aptamer. The structure was predicted by $\mathrm{m}$-fold online server, according to the free-energy minimization algorithm. Color means the probability. existence, due to aptamers were that desorpted from AuNPs bounding to OTA. The obvious color change can be observed by the naked eye, and finer result may be measured by UV-Vis spectra using A650/A520 value. To verify the feasibility of the method, white wine was chosen as the sample to exclude color interference and added OTA was successfully detected accordingly, which indicated that this method was reliable.

\section{Experimental}

\section{Materials and DNA aptamer}

OTA standard was purchased from Pribolab Pte. Tr. Ltd (Singapore). $\mathrm{HAuCl}_{4} \cdot 4 \mathrm{H}_{2} \mathrm{O}$ was purchased from Sinopharm Chemical Reagent Co., Ltd. Sodium citrate and other regents were purchased from Beijing Chemical Works Co., Ltd. All chemicals used were of analytical grade.

The single-strand DNA (ssDNA) aptamer used in the present study was synthesized by Sangong Biotech Co., Ltd. (Shanghai, China). The sequence of the ochratoxin A (OTA) aptamer was 5'-GAT CGG GTG TGG GTG GCG TAA AGG GAG CAT CGG ACA-3' (Fig. 1).

\section{Instrumentation}

Transmission electron microscopy (TEM) observation was carried out with a Hitachi 800 (Hitachi Ltd., Tokyo, Japan). UV-Vis spectra were obtained using a Nanodrop 2000c (Thermo Scientific, Wilmington, USA).

\section{Preparation of AuNPs}

The gold nanoparticles synthesis approach used the classical citrate reduction method. A $100-\mathrm{mL}$ solution of $1 \mathrm{mM} \mathrm{HAuCl}_{4}$ was heated to boiling point with steady stirring, and $3 \mathrm{~mL}$ of $1 \%$ trisodium citrate solution was added to the solution rapidly. The solution continued to boil with mixing for $15 \mathrm{~min}$. The AuNPs sample was cooled to room temperature and stored at $4^{\circ} \mathrm{C}$ in a brown bottle with $13 \mathrm{~nm}$ diameter before use. TEM observation was obtained by a Hitachi 800 at $200 \mathrm{kV}$ (Fig. 2A). The final AuNPs concentration was determined to be $4 \mathrm{nM}$ using a Nanodrop 2000c UV-Vis spectrophotometer based on the extinction measured at $520 \mathrm{~nm}$ (Fig. 2B), with $\varepsilon=2.4 \times 10^{8}$ $\mathrm{L} \mathrm{mol}{ }^{-1} \mathrm{~cm}^{-1}$.

\section{Detection of OTA}

To establish AuNPs based colorimetric aptasensor for OTA detection, the following facile processes were followed. Briefly,
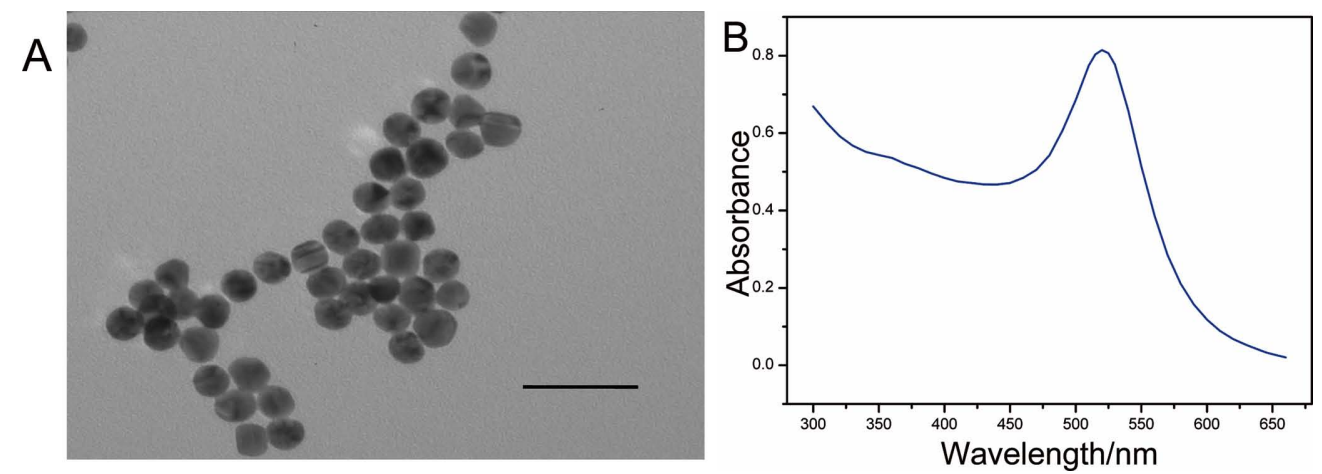

Fig. 2 TEM image and UV-Vis spectrum of the $13 \mathrm{~nm}$ AuNPs. (A) Observation of dispersed AuNPs with magnification of 10000. (B) UV-Vis spectrum of the AuNPs with Nanodrop 2000c UV-Vis spectrophotometer. 
aptamer was dissolved in $1 \mathrm{mM}$ PBS buffer with $0.1 \mathrm{mM} \mathrm{MgCl}$ $40 \mu \mathrm{L}$ of different concentrations of OTA solution mixed with same volume of $0.6 \mathrm{mM}$ aptamer for $15 \mathrm{~min}$ at $4^{\circ} \mathrm{C}$. The mixed solution was added to AuNPs $200 \mu \mathrm{L}$ and allowed to incubate for another $5 \mathrm{~min}$ at room temperature. Then $40 \mu \mathrm{L}$ of $180 \mathrm{mM}$ $\mathrm{NaCl}$ was added to the mixed solution. After incubating for $5 \mathrm{~min}$, the UV-Vis absorption spectrum was measured over the wavelength ranging from 300 to $700 \mathrm{~nm}$.

\section{Treatment of real sample}

In the study $10 \mathrm{~mL}$ white grape wine as real sample was used, due to its transparent and liquid peculiarity, and a simple and

A

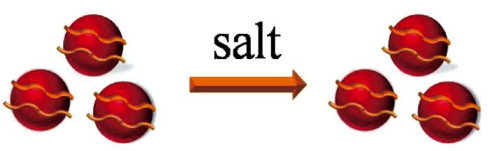

B

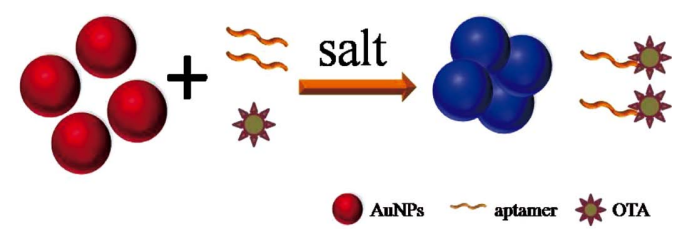

Fig. 3 Schematic illustration of the colorimetric assay for OTA utilizing the specific aptamers and AuNPs. (A) The surface of AuNPs is absorbed by aptamer and this protects AuNPs from aggregation. (B) Aptamer bounding with the existence of OTA desorped from the AuNPs in the system with color changing from red to blue. convenient method was chosen. The ethanol in the white wine was removed by rotary evaporation. Then $3 \mathrm{~mL}$ acetonitrile and distilled water was added to the round bottomed flask. After ultrasonic extraction for $15 \mathrm{~min}$ at room temperature, white grape wine samples with final volume of $10 \mathrm{~mL}$ were analyzed.

\section{Results and Discussion}

Colorimetric detection of OTA by using AuNPs and ssDNA aptamers

In our system, the AuNPs probes were synthesized by the reduction of $\mathrm{HAuCl}_{4}$. AuNPs can maintain a dispersed state based on the repulsion between nanoparticles against the aggregation caused by strong Van der Waals attraction. As is known to all, the surface of gold nanoparticles could be adsorbed by unbounded aptamer inhibiting the aggregation of AuNPs. By reason of the aptamer-target interaction, aptamer desorps from gold nanoparticles then tightly couple OTA existed in the system. ${ }^{24}$ The aggregation of AuNPs is caused by the existence of salt, leading to the solution color changing from red to blue (Fig. 3). By using a spectrophotometer to measure the sample solution, the concentration of OTA was determined with the ratio of the absorbance in wavelength of 520 and $650 \mathrm{~nm}$.

\section{Effect of $\mathrm{NaCl}$ concentration}

The aggregation of AuNPs was affected by the concentration of $\mathrm{NaCl}$ added to the solution. When there is the presence of a concentrations amount of salt, the color changed from red to blue quickly. Moreover, the AuNPs could only remain stable in low concentration of salt and high concentrations could cause
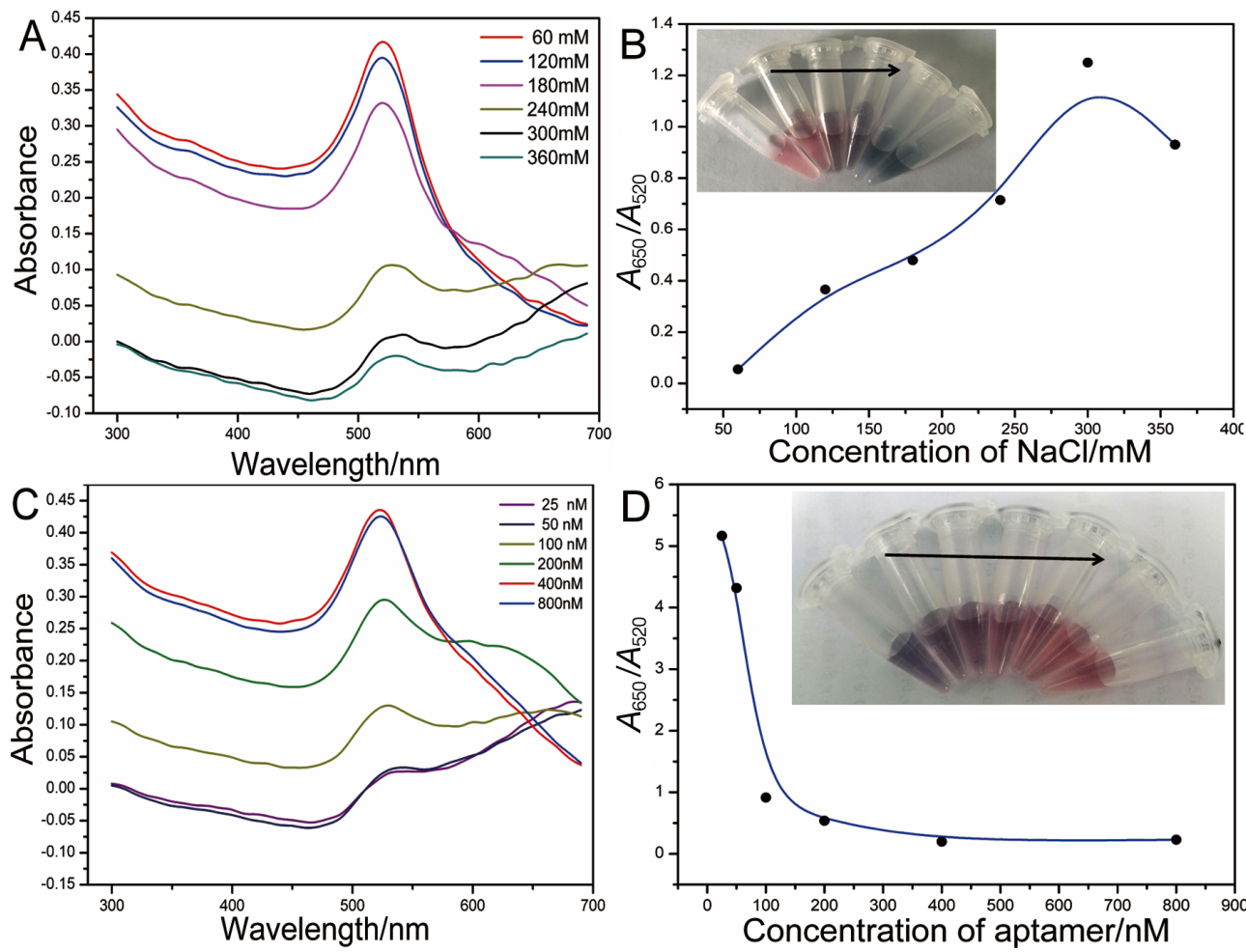

Fig. 4 Optimization of key parameters of the label-free nano-aptasensor. (A) UV-Vis spectra for reactions of various concentration of $\mathrm{NaCl}$. (B) Optimization of concentration of $\mathrm{NaCl}(60,120,180$, 240, 300, $360 \mathrm{mM}$ ). (C) Absorption spectra and corresponding color change of AuNPs with the presence of different concentrations of aptamer. (D) Optimization of concentration of the aptamer $(0,25,50,100,200,400,800 \mathrm{nM})$. 

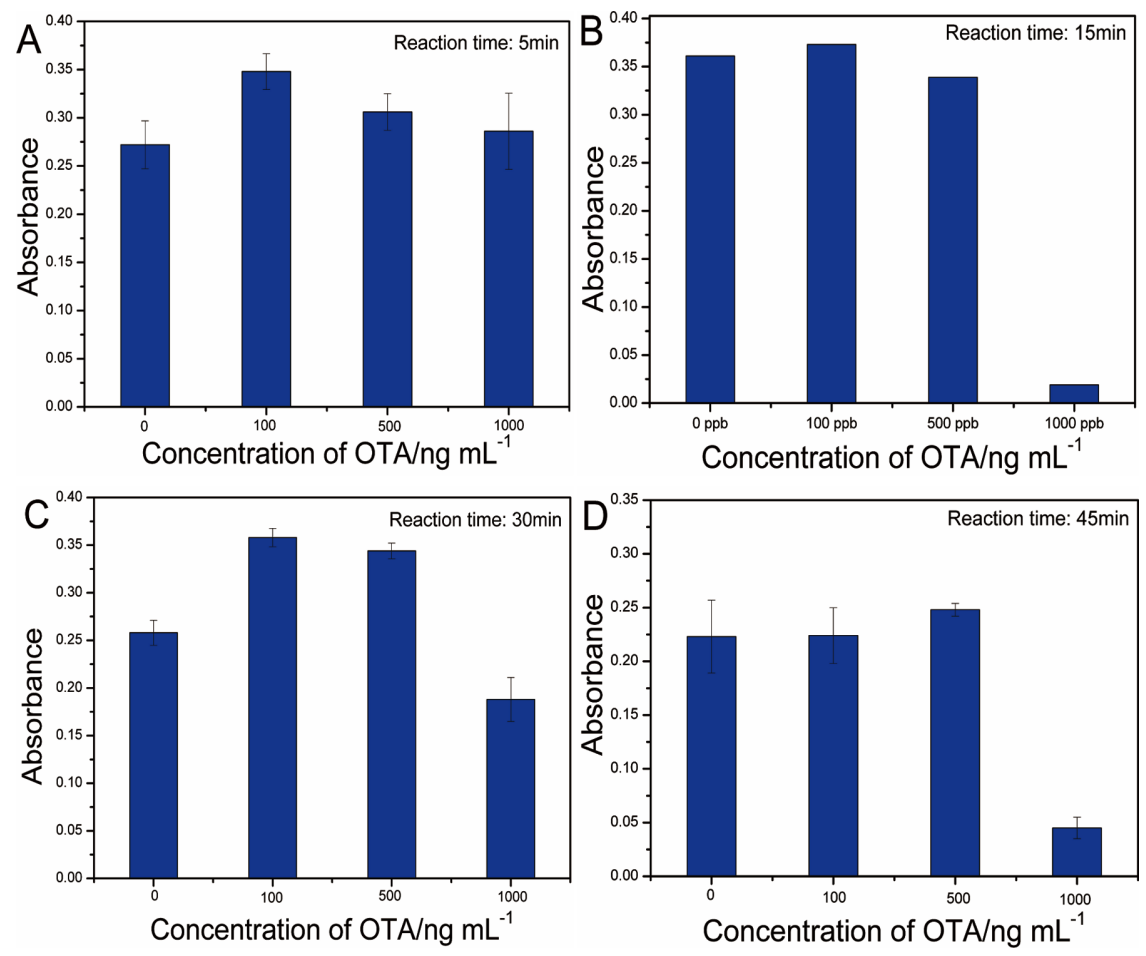

Fig. 5 Variation of $A_{520}$ value of the AuNPs versus the reaction time (5, 15, 30, $\left.45 \mathrm{~min}\right)$ after addition of different concentrations of OTA $(0,100,500,1000 \mathrm{ng} / \mathrm{mL})$.

the aggregation of AuNPs even without the presence of OTA, which might reduce the applicability of the method. Hence, we mixed $40 \mu \mathrm{L}$ different concentrations of $\mathrm{NaCl}$ and $200 \mu \mathrm{L}$ AuNPs for 5 min as detailed in Fig. 4A. When the concentration of $\mathrm{NaCl}$ reached $300 \mathrm{mM}$, the color of AuNPs turned at once as shown in Fig. 4B. The ratio of the absorbance of 520 and $650 \mathrm{~nm}$ achieved peak value when the amount of $\mathrm{NaCl}$ added was $300 \mathrm{mM} ; 300 \mathrm{mM} \mathrm{NaCl}$ was thus the best optimized choice to make the color difference more obvious under the presence and absence of OTA.

\section{Effect of aptamer concentration}

The amount of aptamer was supposed to be the key factor to the sensitivity in the study. A low content of aptamer could not stop the aggregation of AuNPs with the existence of salt because there was not a sufficient amount of aptamer adsorbed on the surface of AuNPs. While excessive aptamer might reduce the sensitivity of the developed system with part of aptamers unbounded. Hence, the appropriate concentration of aptamer can protect AuNPs from aggregation and aptamers were almost all bounded tightly to AuNPs with few of them still in the free state. As is shown in Fig. 4C, the UV-Vis spectra were detected from the wavelength of 300 to $700 \mathrm{~nm}$. And the color change can be obviously observed as Fig. 4D. The ratio of absorbance values of 520 and $650 \mathrm{~nm}$ became stable when $400 \mathrm{nM}$ aptamer was added. From the above, $400 \mathrm{nM}$ aptamer was chosen for further investigation.

\section{Effect of reaction time}

The reaction time affected the binding of OTA and aptamer. To monitor the effect of reaction time, the adsorption measurements were carried out at time values 5 to $45 \mathrm{~min}$ and the selected concentration of OTA at the same time, accordingly. The results showed that the $A_{520}$ value had significant difference at $15 \mathrm{~min}$. When the incubate time was less than $15 \mathrm{~min}$, the binding of OTA and aptamer was quite loose and the aptamerOTA complex was easy to desorb, and the difference of $A_{520}$ value induced by the binding of OTA with aptamer was not obvious as illustrated in Fig. 5A. The difference of $A_{520}$ value became unapparent when reaction time was more than $15 \mathrm{~min}$ as in Figs. 5C and 5D, which was probably because the binding of OTA and aptamer was too tight, and it was difficult for AuNPs to obtain aptamer through competition. Thus, reaction time of 15 min was chosen, and the difference of $A_{520}$ absorbance was quite obvious as in Fig. 5B.

\section{Analytical performance of the assay}

To summarize, the optimized experiment conditions are listed as follows: (a) a $300 \mathrm{mM}$ concentration of $\mathrm{NaCl}$, (b) a $0.4 \mathrm{mM}$ concentration of aptamrers, (c) a 15-min incubation time of OTA with aptamer. With the above optimized parameters, we developed the label-free AuNPs-based colorimetric assay for OTA detection. As illustrated in Fig. 6B, The ratio of the absorbance of 520 and $650 \mathrm{~nm}$ of AuNPs gradually increased with the increment of OTA concentration leading to color changes of AuNPs from red to blue. The variation of the ratio increased linearly with the OTA concentration in the range of $32-1024 \mathrm{ng} / \mathrm{mL}$. The detection limit of the method was calculated as low as $20 \mathrm{ng} / \mathrm{mL}$ as per the scheme of three times standard deviation of blank response. In addition, all detection procedures could be accomplished in approximately $25 \mathrm{~min}$.

\section{Selectivity for the assay}

In order to characterize the association behavior of the OTA aptamer, UV-Vis responses of the aptamer-AuNPs- $\mathrm{NaCl}$ system towards ochratoxin B (OTB) as structural analogue and two mycotoxin chemicals (FB and AFB1) probably coexisting with OTA (Fig. 7), were individually investigated. ${ }^{25}$ As depicted in 

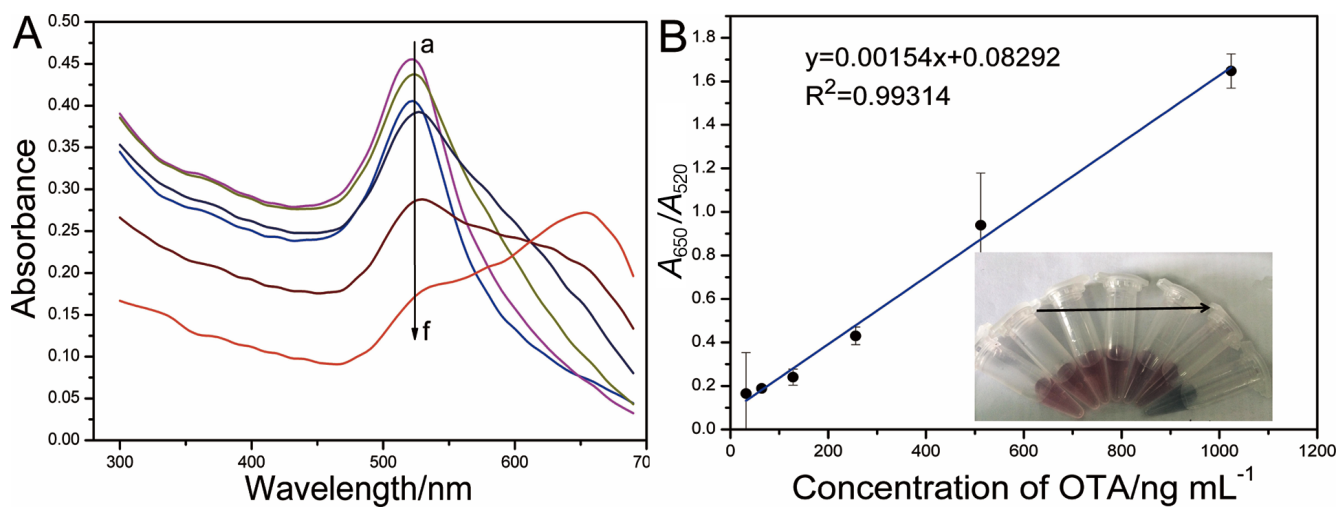

Fig. 6 A colorimetric detection of OTA using OTA binding aptamers and AuNPs. (A) Absorption spectra of the AuNPs when the aptasenor was utilized for different concentrations of OTA (from top to bottom: 32, 64, 128, 256, 512, $1024 \mathrm{ng} / \mathrm{mL}$ ) under the optimized experimental conditions. (B) Absorbance ratio of AuNPs at 520 and $650 \mathrm{~nm}\left(A_{650} / A_{520}\right)$ versus concentration of OTA (inset: corresponding photographic images, the concentration of OTA added from left to right were $0,32,64$, $128,256,512,1024 \mathrm{ng} / \mathrm{mL})$.

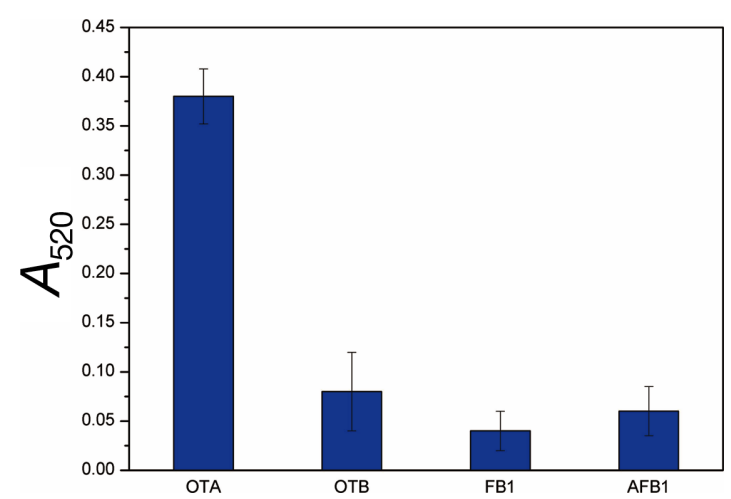

Fig. $7 A_{520}$ of AuNPs in the presence of OTB and several probably coexisting chemicals $(200 \mathrm{ng} / \mathrm{mL})$.

Fig. 7, the absorbance value of AuNPs with the presence of OTA is much higher than that of OTB, FB, and AFB1. The results indicated that the aptamer-based sensor exhibited excellent selectivity towards OTA. ${ }^{26}$

\section{Application in real sample}

In order to ensure the feasibility of the present method in practical applications, the detection of OTA in white wine was carried out. The practical samples were fortified with certain amounts of OTA. Table 1 shows that the recoveries of the whole samples are in the range of 100.80 to $112.50 \%$. The desirable recoveries demonstrate the reliability of the developed method for detection of OTA in real world samples.

\section{Conclusions}

In summary, a successfully sensitive, accurate method was developed by using unlabeled aptamer-based colorimetric biosensor for the determination of OTA based on free gold nanoparticles without complicated modification and expensive instruments. The red to blue color change in the presence of OTA could be easily observed by the naked eye or measured by
Table 1 Determination of OTA in white wine samples $(n=3)$

\begin{tabular}{ccccc}
\hline $\begin{array}{c}\text { White wine } \\
\text { sample }\end{array}$ & $\begin{array}{c}\text { Level added/ } \\
\text { ng mL }\end{array}$ & $\begin{array}{c}\text { Amuont } \\
\text { found/ } \\
\text { ng mL }\end{array}$ & $\begin{array}{c}\text { Recovery, } \\
\%\end{array}$ & $\begin{array}{c}\text { RSD, } \\
\%\end{array}$ \\
\hline 1 & 60 & 64.787 & 107.97 & 2.05 \\
2 & 120 & 130.64 & 108.87 & 1.92 \\
3 & 480 & 540.04 & 112.50 & 4.27 \\
4 & 960 & 968.25 & 100.80 & 1.43 \\
\hline
\end{tabular}

using a UV-Vis spectrometer. By optimizing the three key factors, i.e. the concentration of $\mathrm{NaCl}$, the amount of aptamer, and the time of the binding of OTA and aptamer, the linear range and detection limit were found to be 32 to $1024 \mathrm{ng} / \mathrm{mL}$ and $20 \mathrm{ng} / \mathrm{mL}$, respectively. The result for the identification of OTA can be easily affirmed by the naked eye. More importantly, the proposed method has been successfully applied to the rapid detection of OTA in white wine samples with less time. The aptamer-based colorimertic method would be a promising rapid detection strategy for OTA, and can be also used for the fast screening of other food contaminants.

\section{Acknowledgements}

This research was supported by Beijing "Thirteen-Five" food safety research on the key technology and realization of path (Grant No. Z141108001314016) together with the Key Program of a Synchronized Detection Research and Risk Assessment for Radionuclides in Foods of Beijing Municipal Science \& Technology Commission (D161100002116002).

\section{References}

1. A. Rhouati, C. Yang, A. Hayat, and J. L. Marty, Toxins, 2013, 5, 1988.

2. L. Yang, Z. Ying, R. Li, C. Lin, L. Guo, B. Qiu, Z. Lin, and G. Chen, Biosens. Bioelectron., 2015, 70, 268.

3. W. Ma, H. Yin, L. Xu, Z. Xu, H. Kuang, L. Wang, and 
C. Xu, Biosens. Bioelectron., 2013, 42, 545.

4. J. Chen, Z. Xi, S. Cai, D. Wu, C. Mei, S. Wang, and J. Zhang, Biosens. Bioelectron., 2014, 57C, 226.

5. H. P. van Egmond, R. C. Schothorst, M. A. Jonker, and Anal. Bioanal. Chem., 2007, 389, 147.

6. R. Liu, Y. Huang, Y. Ma, S. Jia, M. Gao, J. Li, and H Zhang, ACS Photonics, 2015, 7, 6982.

7. X. Yang, W. Kong, Y. Hu, M. Yang, L. Huang, M. Zhao, and Z. Ouyang, J. Sep. Sci., 2014, 37, 853.

8. Y. Zheng, Y. Wang, and X. Yang, Sens Actuators, B, 2011, 156, 95.

9. J. Chen, Z. Li, J. Ge, R. Yang, L. Zhang, L. B. Qu, H. Q. Zhang, and L. Zhang, Talanta, 2015, 139, 226.

10. L. Barthelmebs, A. Hayat, A. W. Limiadi, J. L. Marty, and T. Noguer, Sens, Actuators, B, 2011, 156, 932.

11. J. Zhang, X. Zhang, G. Yang, J. Chen, and S. Wang, Biosens. Bioelectron., 2013, 41, 704.

12. A. Rhouati, C. Yang, A. Hayat, and J. L. Marty, Toxins, 2013, 5, 1988.

13. Y. Luo, J. Xu, Y. Li, H. Gao, J. Guo, F. Shen, and C. Sun, Food Control, 2015, 54, 7.

14. C. Luo, RSC Adv., 2014, 5, 10994.

15. M. R. Hormozi-Nezhad and S. Abbasi-Moayed, Talanta,
2014, 129, 227.

16. Z. Chen, Y. Hu, Q. Yang, C. Wan, Y. Tan, and H. Ma, Sens. Actuators, B, 2015, 207, 277.

17. C. Jing, Z. Li, G. Jia, Y. Ran, Z. Lin, L. B. Qu, and L. Zhang, Talanta, 2015, 139, 226.

18. S. Cai, L. Xin, C. Lau, and J. Lu, Analyst, 2010, 135, 615.

19. C. Yang, Y. Wang, J.-L. Marty, and X. Yang, Biosens. Bioelectron., 2010, 26, 2724.

20. D. Zhao, C. Chen, L. Lu, F. Yang, and X. Yang, Sens. Actuators, B, 2015, 215, 437.

21. M. R. Hormozi-Nezhad and S. Abbasi-Moayed, Talanta, 2014, 129, 227.

22. J. Hu, P. Ni, H. Dai, Y. Sun, Y. Wang, S. Jiang, and Z. Li, Analyst, 2015, 140, 3581.

23. S. Wang, S. Gao, S. Sun, Y. Yang, Y. Zhang, J. Liu, and Y. Dong, RSC Adv., 2016, 6, 45651.

24. Y. S. Kim, J. H. Kim, I. A. Kim, S. J. Lee, J. Jurng, and M. B. Gu, Biosens. Bioelectron., 2010, 26, 1644.

25. C. Wang, J. Qian, K. Wang, X. Yang, Q. Liu, and N. Hao, Biosens. Bioelectron., 2016, 77, 1183.

26. A. T. A. Gomes, D. Paredes, and F. Valentin, Procedia Computer Science, 2011, 4, 688. 